\title{
Well-dressed states for wave-packet dynamics in cavity QED
}

\author{
D. W. Vernooy and H. J. Kimble \\ Norman Bridge Laboratory of Physics 12-33, California Institute of Technology, Pasadena, California 91125
}

(Received 5 February 1997; revised manuscript received 23 June 1997)

\begin{abstract}
The quantization of atomic center-of-mass motion is considered within the context of cavity QED with particular emphasis on the dynamics of localized wave packets. "Well-dressed" states are introduced as an eigenbasis that incorporates both the quantized atom-field interaction and the external bound states of a potential well. The interplay of internal and external time scales generates qualitatively new dynamics such as novel "collapses" and "revivals."' [S1050-2947(97)10411-5]
\end{abstract}

PACS number(s): 42.50.Md, 33.80.Ps, 42.50.Fx

\section{INTRODUCTION}

The phenomenal rate of development of atomic cooling and trapping has important implications for cavity quantum electrodynamics (cavity QED) [1] with cold atoms. For example, recently the first steps have been taken to explore coupled external center-of-mass (c.m.) and internal (atomic dipole+quantized cavity field) dynamics in an experiment involving cold atoms falling one by one through a microcavity with mean intracavity photon number $\leqslant 1$ [2]. Motivated by such emerging technical capabilities and by the potentially broad scientific impact, here we investigate the structure and dynamics for an atom strongly coupled to a quantized field in a regime for which the dipole coupling energy $\hbar g$ is comparable to the atomic kinetic energy $E_{k}$. With cold atoms, a fully quantized treatment of the atomic c.m. degrees of freedom is necessary in order to account for the wavepacket nature of the c.m. state. This situation has not been important for previous experimental work in cavity QED in which atoms from a thermal beam transit through a quantized cavity field with kinetic energy $E_{k}$ large compared to the coupling energy $\hbar g$ [1] and for which the c.m. motion could be treated classically.

That the atom-field coupling for the internal degrees of freedom could have important consequences for the external c.m. motion was recognized in the seminal work of Refs. [3-6], where scattering from the localized potential $\hbar g(\mathbf{r})$ as well as trapping was first considered. Since then, a number of treatments of quantized motion in cavity QED have appeared related to diverse problems in optical physics, including cooling and trapping in a cavity with $n \sim 1$ quanta $[4,7,8]$, atomic position measurements via entanglement with the cavity field [9-11], and quantum nondemolition detection of the intracavity photon number based upon atomic deflections [12-15]. Also analyzed have been the role of atomic motion on the intracavity radiative process, as, for example, in Refs. [16,17], and other progeny in cavity QED, including most importantly the recent work on the micromaser with quantized c.m. motion [18].

Within the context of this general theme, our current analysis explores the interplay of the finite spatial extent of a c.m. wave packet $\psi(\mathbf{r})$ with the quantum field mode structure $g(\mathbf{r})$, as is relevant to several important areas, including quantum logic with atoms in a cavity $[19,20]$ and quantum state synthesis in cavity QED [21,22].
Beginning in Sec. II with the spectrum of eigenvalues, we extend the familiar dressed states of the Jaynes-Cummings Hamiltonian to include bound c.m. states that arise either because of an intrinsic spatial variation in the atom-field coupling $g(\mathbf{r})$ or because of an externally applied atomic potential $V_{\text {ext }}(\mathbf{r})$, e.g., as in an rf Paul trap [23]. Spatially localized eigenstates for both the external motion in a potential well and for the internal atom-field interaction are termed "welldressed" states, with the eigenvalue spectra in three distinct regimes illustrated in Fig. 1 and described in more detail below.

To explore the type of new dynamical phenomena that can arise from the interplay of internal and external degrees of freedom, we consider in Sec. III an atom initially localized in the mode $g(\mathbf{r})=g_{0} U(\mathbf{r})$ with one photon and find substantial modifications to the familiar Rabi nutation rate $2 g(\mathbf{r})$. New coupling strengths $g_{\text {eff }}^{(1,2)}$ characterize the initial

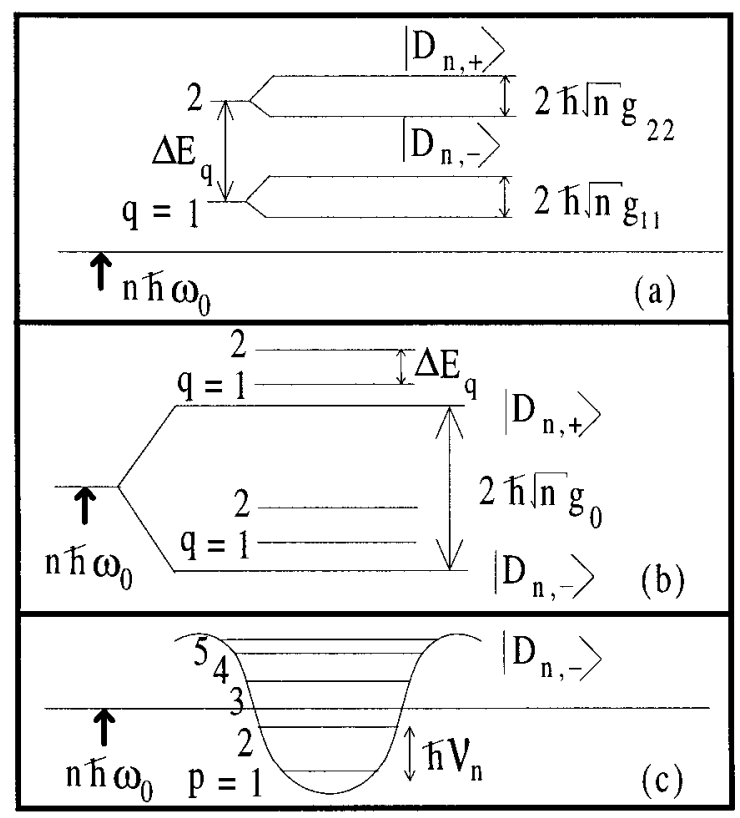

FIG. 1. Eigenvalue spectra for the "well-dressed" states. Shown is the $n$-excitation manifold for the three cases (a) $V_{\text {ext }} \gg \hbar g_{m} \sqrt{n}$, (b) $\hbar g_{m} \sqrt{n} \gg V_{\text {ext }}$, and (c) $V_{\text {ext }}=0$, where in (c), the curvature in $g(\mathbf{r})$ supports bound states. The quantities $\mathcal{V}_{n}$ and $g_{q q} \equiv\left\langle\psi_{q}|g(\mathbf{r})| \psi_{q}\right\rangle$ are discussed in the text. 
evolution of the atomic dipole and inversion, which are in general distinct quantities with a strong dependence on the atomic c.m. state, being given by overlap integrals of $g(\mathbf{r})$ and the c.m. wave functions $\psi(\mathbf{r})$. More generally, already implicit in Fig. 1 for the eigenvalues of the well-dressed states are new c.m.-dependent spatial and temporal scales, $\tau_{i}$, where for $g_{0} \tau_{1} \gtrsim 1$, wave-packet delocalization can lead to a "collapse" of internal atom-field coherences while for $g_{0} \tau_{2}>1$ "revivals" of this coherence are possible.

An emphasis on localized bound states distinguishes our work from most previous treatments of quantized motion in cavity QED [4-19], which have largely dealt with scattering of unbound momentum eigenstates. Perhaps the treatments of most relevance to our own current effort is the work of Refs. [17,18]. In Ref. [17], for example, a quantum mechanical treatment of an atom in a standing wave optical cavity is used to study the diffusion in momentum space of an initial c.m. momentum eigenstate and the modifications of the radiative spectra due to c.m. quantization. Although a special case of Fig. 1(c) in this present paper was introduced in Ref. [17] for a one-dimensional (1D) standing wave, the formalism is applied there to delocalized momentum states that extend over many wavelengths (e.g., see Figs. 1-3 of Ref. [17]). By contrast, our emphasis is on the structure and dynamics of spatially localized bound states (such as those formed in the potential seen by an atom confined to a single optical wavelength) for which the dynamical processes have not been previously considered. These localized wave functions are complex superpositions of (delocalized) Bloch states and, for the deepest bound states we consider here, a typical tunneling time would be by far the longest time scale in the problem.

Bound-state structure does play a critical role in the beautiful treatment in Ref. [18] of the "mazer," for which incident cold atoms are transmitted or reflected from the quantized field of a micromaser. In this work, the resonances in the scattering process are associated with the uppermost bound state in the potential formed by the quantized field. This treatment, however, tended not to emphasize the size scale of the initial c.m. wave packet with respect to the typical wavelength of the quantum field nor the dynamics within the potential well itself. The analyses in $[15,16]$, though very interesting in their own right, also do not place the same emphasis on localized c.m. wave packets or their expansion in terms of the bound states of the optical potential as we do here.

Note that all this work, including our own, builds to some extent on preceding work with classical fields outside the realm of cavity QED in which both internal and external (c.m.) atomic degrees of freedom must be quantized in the presence of resonant or quasiresonant excitation. Optical molasses (including treatments of delocalized momentum states) [24], trapped ions [25,26], atom lasers [27], and novel atomic cooling and trapping schemes [28] using classical light fields and their associated forces have all been treated in this way.

In such problems involving atoms, the Raman-Nath approximation is often invoked, which tends to deemphasize the motion of the atom over its internal state lifetime. For ions, especially given the recent experimental interest in coupling ion traps with high-finesse optical cavities, one must consider dynamics when the Lamb-Dicke parameter is large enough to spread the c.m. wave packet over an appreciable fraction of an optical wavelength. These are things that we wish to allow for here, contingent upon the relative magnitudes of the typical energies in the problem, as will be developed further in Sec. II.

Against this backdrop, the structure of the present paper is as follows. In Sec. II we explore the structure of the welldressed states in three distinct regimes. In Sec. III, dynamics in two of these regimes is investigated. A simple 1D model is used to illustrate some of the unique features that can arise within our formalism. Finally, Sec. IV includes a brief discussion of the role of dissipation on the otherwise coherent dynamics of Sec. III.

\section{STRUCTURE OF THE WELL-DRESSED STATES}

Turning now to the details of our treatment, we begin by introducing the system Hamiltonian

$$
H=\frac{\mathbf{p}^{2}}{2 m_{a}}+V_{\mathrm{ext}}(\mathbf{r})+\hbar g(\mathbf{r}) H_{I F},
$$

where $H_{I F}=\left(\sigma_{-} a^{\dagger}+a \sigma_{+}\right)$is the Jaynes-Cummings interaction written in a rotating frame at $\omega_{\text {atom }}=\omega_{\text {cavity }} \equiv \omega_{0}$. Here, $\mathbf{r}, \mathbf{p}$ are c.m. position and momentum operators for an atom of mass $m_{a}, \sigma_{+}$is the internal atomic raising operator, and $a^{\dagger}$ is the creation operator for the cavity field mode. $V_{\text {ext }}$ is assumed to have a bound-state structure $\left\{\left|\psi_{q}\right\rangle, E_{q}\right\}$ when $g(\mathbf{r})=0$, and similarly, the term in $g(\mathbf{r})$ can be interpreted as an internal state-dependent potential where, for $V_{\text {ext }}=0$, we have eigenstates $\left\{\left|\phi_{p}\right\rangle, E_{p}\right\}$ of $H$. The well-known dressed states of $H_{I F}$ are [29]

$$
\left|D_{n, \pm}\right\rangle=\frac{1}{\sqrt{2}}(|e, n-1\rangle \pm|g, n\rangle),
$$

with $E_{n, \pm}= \pm \sqrt{n}$ and with the convention that $g(\mathbf{r})>0[g(\mathbf{r})<0]$ corresponds to a decrease (increase) in energy for $\left|D_{n,-}\right\rangle\left(\left|D_{n,+}\right\rangle\right)$. For the time being, we neglect the effects of dissipation.

Rather than specify a particular form for $g(\mathbf{r})$, we concentrate first on the generic behavior of $g(\mathbf{r})$ around a field extremum $g\left(\mathbf{r}_{0}\right)$. For simplicity, we consider one dimension with $\mathbf{r}_{0} \rightarrow z_{0}$ and with $g_{m} \equiv g\left(z_{0}\right)$. Here, $\left.\xi \equiv\left|\left(1 / g_{m}\right)\left[d^{2} g(z) / d z^{2}\right]\right|_{z=z_{0}}\right|^{-1 / 2}$ characterizes the magnitude of the curvature of $g\left(z_{0}\right)$, with $\eta$ its sign. In a harmonic approximation, the bound states $\left\{\left|\phi_{p}\right\rangle\right\}$ associated with the dressed state $\left|D_{n,-}\right\rangle$ (in the case of a single field maximum with $\eta<0)$ have an energy spectrum $E_{p}=\left(p+\frac{1}{2}\right) \hbar \mathcal{V}_{n}$ $(p=0,1,2, \ldots)$, where

$$
\hbar \mathcal{V}_{n}=\sqrt{2\left(\hbar g_{m} \sqrt{n}\right)\left(\frac{\hbar^{2}}{2 m_{a} \xi^{2}}\right)} .
$$

In general, the requirement for bound states is that $\sqrt{2 R_{n}}<1$, with

$$
R_{n} \equiv \frac{\left(\hbar^{2} / 2 m_{a} \xi^{2}\right)}{\hbar g_{m} \sqrt{n}}
$$


Note that $R_{1}$ is analogous to the parameter $R$ in [17].

As our intention here is to identify generic features brought by the c.m. state dependence to the usual dressedstate structure, we will not consider the general solution of Eq. (1) with arbitrary functional forms for $g(\mathbf{r})$ and $V_{\text {ext }}(\mathbf{r})$. Instead, with the straightforward definitions and observations above, we can understand the bound-state structure of $H$ in several limiting cases of experimental relevance. The three energy scales necessary for determining the appropriate c.m. eigenbasis are the coupling energy $\hbar g_{m} \sqrt{n}$, the energy splitting $\Delta E_{q}$ for the bound states $\left\{\left|\psi_{q}\right\rangle\right\}$ of $V_{\text {ext }}$, and the energy splitting $\hbar \mathcal{V}_{n}$ associated with the bound states $\left\{\left|\phi_{p}\right\rangle\right\}$ of $\hbar g(\mathbf{r})$.

Perhaps the simplest case to consider first is that for which the external potential is dominant, namely, $\Delta E_{q} \gg \hbar g_{m} \sqrt{n}$. In this regime, the well-dressed state structure is solved using $\left\{\left|\psi_{q}\right\rangle\right\}$ as a fixed basis for the c.m. and $\hbar g(\mathbf{r}) H_{I F}$ is viewed as a perturbation. The eigensolutions of $H$ are $\left\{\left|\psi_{q}\right\rangle \otimes\left|D_{n, \pm}\right\rangle, E_{q} \pm \hbar \sqrt{n} g_{q q}\right\}$, where the matrix element $g_{q q}$ is defined by

$$
g_{q q} \equiv\left\langle\psi_{q}|g(\mathbf{r})| \psi_{q}\right\rangle=\langle g(\mathbf{r})\rangle_{q} .
$$

The finite size of the wave packet inherent in $g_{q q}$ distinguishes this case from much of the previous work in cavity QED. It can certainly be the case that $\langle g(\mathbf{r})\rangle_{q} \neq g\left(\langle\mathbf{r}\rangle_{q}\right)$ for the bound states of $V_{\text {ext }}$, as in Figs. 2(a), and 2(b). One sees that this condition arises when the atomic c.m. wave function has spatial structure on a scale commensurate with that of the quantum field to which it is coupled. Variations in atomic wave-packet probability density on this scale can profoundly influence the coupled atom-field evolution.

More generally, the c.m.-dependent Jaynes-Cummings ladder (i.e., the well-dressed states) for this regime is illustrated in Fig. 1(a). Note that "Rabi flopping" proceeds at the c.m. state-dependent rate $2 \sqrt{n} g_{q q}$, which can be completely suppressed $\left(g_{q q} \rightarrow 0\right)$ due to the spatial structure of $g(\mathbf{r})$ with respect to $\psi_{q}(\mathbf{r})$. Spectroscopic investigation of the structure would, in general, involve c.m. state-dependent transitions within each manifold. With reference to Fig. 1(a), for example, a transition from $\left|\psi_{q}\right\rangle \otimes\left|D_{n,-}\right\rangle$ to $\left|\psi_{q^{\prime}}\right\rangle \otimes\left|D_{n,+}\right\rangle$ would have its amplitude determined by the value of its c.m. structure-dependent "Franck-Condon" factor $g_{q^{\prime} q}$ $=\left\langle\psi_{q^{\prime}}|g(\mathbf{r})| \psi_{q}\right\rangle$.

A second regime interchanges the roles of $V_{\text {ext }}(\mathbf{r})$ and $\hbar g(\mathbf{r})$, such that $\hbar g_{m} \sqrt{n} \gg \Delta E_{q} \gg \hbar \mathcal{V}_{n}$. Here, $\xi$ is assumed sufficiently large so that the coupling $g(\mathbf{r}) \sim g_{0}$ acts simply as a global shift to $\left\{E_{q}\right\}$. In this regime, the well-dressed states of $H$ are $\left\{\left(\left|\psi_{q}\right\rangle \otimes\left|D_{n, \pm}\right\rangle\right),\left( \pm \hbar \sqrt{n} g_{0}+E_{q}\right)\right\}$ as illustrated in Fig. 1(b). The large curvature $\xi$ in the cavity field allows the atomic c.m. to remain coupled predominantly to the bound states of $V_{\text {ext }}(\mathbf{r})$. The interaction energy associated with the internal degrees of freedom appear more or less as in the standard Jaynes-Cummings ladder, modified now by the fine structure associated with the atomic c.m. In fact there are additionally small shifts $g_{q q}$ of each eigenstate due to the spatial dependence of $g(\mathbf{r})$. These shifts are assumed to be of higher order and are not shown. Note that the separate limits of Figs. 1(a), and 1(b) begin to converge as the bound state spacings of $g(\mathbf{r})$ and $V_{\text {ext }}(\mathbf{r})$ approach one another, opening the way for more complicated structure (as, for ex-

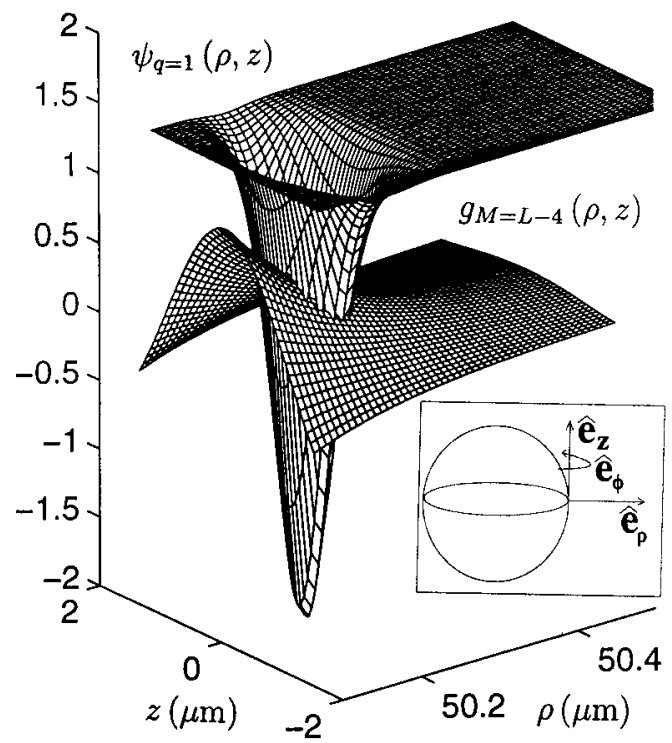

(a)

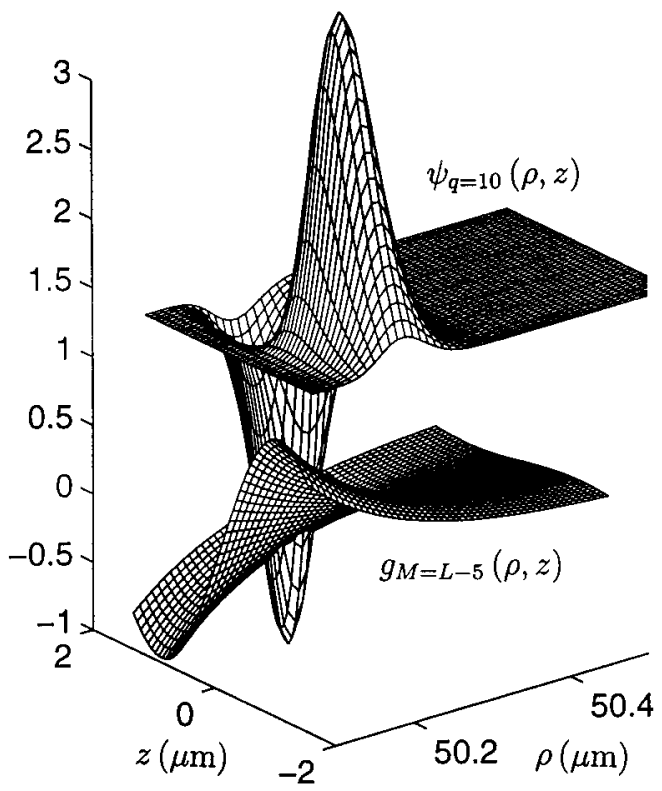

(b)

FIG. 2. Comparison of the c.m. bound states (a) $\psi_{q=10}(\mathbf{r})$ and (b) $\psi_{q=10}(\mathbf{r})$ with the coupling coefficient $g(\mathbf{r})$ for the whispering gallery mode of a fused-silica microsphere of radius $50 \mu \mathrm{m}$ and mode numbers (a) $(L=521, M=517)$ and (b) $(L=521, M=516)$. A dipole-force trap of depth $2 \mu \mathrm{K}$ provides $V_{\text {ext }}$ (the atom gallery of [30]), with $\left\{\psi_{q}(\mathbf{r})\right\}$ determined in [31]. Note in (b) $g_{\text {eff }}^{(1)}=0$ while $g_{\text {eff }}^{(2)} \neq 0$.

ample, with increasing $n$ ) and dynamics. For example, the "vacuum Rabi", transition from the $n=0$ to the $n=1$ manifold must also include the relevant "Franck-Condon" matrix element governing the possibility of an associated c.m. state transition. 
Finally, a third regime takes $\hbar g_{m} \sqrt{n} \gg \hbar \mathcal{V}_{n} \gg \Delta E_{q}$. In fact, here we consider $V_{\text {ext }}(\mathbf{r})$ only as a means of providing a well-defined initial state for an atomic wave packet, after which its effect is assumed negligible compared to the cavity field [e.g., $V_{\text {ext }}(\mathbf{r})$ switched off with $g(\mathbf{r})$ switched on]. For a given $n$ manifold, the Jaynes-Cummings ladder in the dressed-state picture is not split by $\pm \hbar g$. Instead, the welldressed states $\left\{\left|\phi_{p}\right\rangle\right\}$ are associated with the minima of the respective potentials $V_{ \pm}(\mathbf{r})= \pm \hbar \sqrt{n} g(\mathbf{r})$ for $\left|D_{n, \pm}\right\rangle$ as in Fig. 1(c) for the bound states of $V_{-}$, with the repulsive barrier seen by $\left|D_{n,+}\right\rangle$ at that spatial location omitted. This is a consequence of the fact that the dressed state $\left|D_{n,-}\right\rangle$ is attracted towards the regions of negative curvature in $g(\mathbf{r})$ $[\eta<0]$ while $\left|D_{n,+}\right\rangle$ is repulsed, and conversely for $\eta>0$. This structure is heavily dependent on well depth and changes with the manifold level $n$. Nevertheless, we emphasize that in the harmonic approximation (and indeed for more general potentials as well) the level spacing within a given $n$-manifold is given by $\hbar \mathcal{V}_{n}$ and scales as $(n)^{1 / 4}$. Note that this same factor $(n)^{1 / 4}$ plays a central role in the work of [18], for scattering of a cold atom from a potential formed by the cavity field. In that case, $\xi$ is set by the length $L$ of the well and successive scattering resonances for increasing $g_{0} L$, in the low-energy limit, are associated with increments of the round trip phase in units of $2 \pi$, which is precisely the condition for the addition of another bound state in the associated square well potential of depth $g_{0}$. Implicit in that analysis is the use of an external mechanism, such as the potential $V_{\text {ext }}(\mathbf{r})$, to produce the initially cold wave packet.

In terms of a physical implementation, an example relevant to the two regimes of Fig. 1(a) and 1(b) is a trapped ion interacting with a cavity mode. In the microwave domain [1], typical cavities have $g_{0} / 2 \pi \sim 20 \mathrm{kHz}$, while the vibrational frequencies for an rf Paul trap acting as $V_{\text {ext }}(\mathbf{r})$ are $\Delta E_{q} / h \sim 1 \mathrm{MHz}$ and $\xi \ll \lambda_{\mu \text {-wave, }}$, so that the conditions for Fig. 1(a) are satisfied. By contrast, in the optical domain $[1,2] g_{0} / 2 \pi \sim 10 \mathrm{MHz}$, as is appropriate to Fig. 1(b), where for a heavy atom, $\mathcal{V}_{n} / 2 \pi \sim 200 \mathrm{kHz} \leqslant \Delta E_{q} / h$ in an optical standing wave.

For the regime of Fig. 1(c), we have in mind a light atom such as $\mathrm{He}^{*}$, initially prepared in a c.m. eigenstate of $V_{\text {ext }}(\mathbf{r})$, which could be switched on in the form of a dipoleforce trap as in Ref. [32] to provide an initial set of welldefined eigenstates with $g(\mathbf{r})=0$. The atom is then allowed to interact with a single mode of an optical cavity. This requires a transition to $V_{\text {ext }}(\mathbf{r})=0$ with $g(\mathbf{r}) \neq 0$ and could be accomplished for $V_{\text {ext }}(\mathbf{r})$ by simply gating the intensity of the dipole-force trap, while $g(\mathbf{r})$ could be controlled via the mechanism discussed in Refs. [21,22,20]. Here, the effective coupling is given as the geometric mean of $g(\mathbf{r})$ and an external control field $\Omega(t)$, with the "two-level" atom being the two ground states in a $\Lambda$ configuration connected via the excited state by $g(\mathbf{r})$ and $\Omega(t)$. Some realistic experimental parameters in the regime of Fig. 1(c) for $\mathrm{He}^{*}$ will be discussed in Sec. IV.

\section{DYNAMICS}

Rather than focus on any one of the diverse applications of the eigenvalue structure of Fig. 1, we turn to a discussion of dynamics in an attempt to emphasize some of the generic features encountered for wave-packet dynamics in a quantized cavity field. We begin with atomic spontaneous emission in the regime of Fig. 1(a). Here we have utilized the well-dressed states for an atom bound near the surface of a dielectric microsphere (as in Fig. 2) to calculate modifications of radiative processes including the c.m. state and have found significant $(100 \%)$ state-to-state variations in the spontaneous decay rates associated with the finite extent of $\psi_{q}(\mathbf{r})$ as compared to $g(\mathbf{r})$ via $g_{q q}$. Expressed in qualitative terms, this work extends the well-known results for altered radiative processes for a point dipole in the vicinity of a dielectric boundary to the case of an extended wave packet. As well, the dynamics accompanying heating of the atomic c.m. motion in the atom gallery [30] depend strongly on $\psi_{q}(\mathbf{r})$, with sharply decreasing rates of heating with increasing $q$, as discussed in detail in [31].

To address more directly issues of wave-packet dynamics in cavity QED in a nonperturbative regime (i.e., coherent coupling $\gg$ cavity or atomic dissipative rates), we now concentrate on a simple example in the regime appropriate to Fig. 1(c). For an initial c.m. wave packet $\left|\Psi_{\text {c.m. }}(0)\right\rangle$ $=\int d^{3} \mathbf{r} f_{0}(\mathbf{r})|\mathbf{r}\rangle$ and a coupled field and internal atomic state $\left(c_{+}\left|D_{n,+}\right\rangle+c_{-}\left|D_{n,-}\right\rangle\right)$, we find the full quantum state at any later time $t$ to be

$$
\left|\Psi_{\text {tot }}(t)\right\rangle=\sum_{\epsilon= \pm}\left(c_{\epsilon} e^{-(i / \hbar) H_{\epsilon} t}\left|D_{n, \epsilon}\right\rangle\right)\left|\Psi_{\text {c.m. }}(0)\right\rangle
$$

with $H_{ \pm}=\mathbf{p}^{2} / 2 m_{a} \pm \hbar \sqrt{n} g(\mathbf{r})$ and with dissipation neglected. The usual c.m. state $f_{0}(\mathbf{r})=\delta^{3}\left(\mathbf{r}-\mathbf{r}_{0}\right)$ leads to Rabi oscillations with frequency $\Omega_{n}=2 \sqrt{n} g\left(\mathbf{r}_{0}\right)$. However, the wave-packet nature of the c.m. gives rise to new issues that are illustrated in the following 1D problem with the atom initially confined in some $V_{\text {ext }}$, which is quickly switched off (so that $\left|\Psi_{\text {c.m. }}(0)\right\rangle$ cannot adiabatically follow the change). The atom is then coupled to a resonant cavity with mode function $g(z)$. As it is known that the preeminent requirement for possible experimental implementation of wave packet dynamics in cavity QED is that $\hbar g \sim E_{k}$ [4-18], here we assume that the bound state energies of $V_{\text {ext }}$ are initially of the same order as $\hbar g_{0}$ so that an atom prepared in one of these states will remain localized when coupled to the cavity.

For $g t \ll 1$, it is possible to find analytical expressions for the atomic dipole $\left\langle\sigma_{-}(t)\right\rangle$ and the excited state probability $P_{e}(t)=\left\langle\sigma_{+}(t) \sigma_{-}(t)\right\rangle$. For an atom initially in the ground state and $n+1$ quanta in the field, $\left|\Psi_{\text {tot }}(t=0)\right\rangle=|g, n+1\rangle$ $\otimes\left|\Psi_{\text {c.m. }}(0)\right\rangle \quad$ (i.e., $\left.\quad c_{ \pm}= \pm 1 / \sqrt{2}\right)$, we have $\left\langle\sigma_{-}(t)\right\rangle$ $=-i \sqrt{n} g_{\text {eff }}^{(1)} t$, where

$$
g_{\mathrm{eff}}^{(1)} \equiv \int d z g(z)\left|f_{0}(z)\right|^{2}
$$

This reproduces the usual expression

$$
\left\langle\sigma_{-}(t)\right\rangle=-i \cos (\sqrt{n} g t) \sin (\sqrt{n} g t) \sim-i \sqrt{n} g t
$$

for small $t$ if $g \rightarrow g_{\text {eff }}^{(1)}$. Furthermore, $P_{e}(t)=n\left(g_{\text {eff }}^{(2)}\right)^{2} t^{2}$ with

$$
g_{\text {eff }}^{(2)} \equiv \int d z|g(z)|^{2}\left|f_{0}(z)\right|^{2},
$$


which is the small $t$ equivalent of the Rabi probability $\sin ^{2}(\sqrt{n} g t)$ with $g \rightarrow g_{\text {eff }}^{(2)}$. Note that the dependence of $\left\langle\sigma_{-}(t)\right\rangle$ and $P_{e}(t)$ on $f_{0}(z)$ can be profound and unlike anything that a "billiard ball" model for the atomic c.m. might predict. For example, for the particular atom gallery state shown in Fig. 2(b) interacting with the whispering gallery mode (WGM) $g_{M=L-5}, g_{\text {eff }}^{(1)}=0$ so that $\left\langle\sigma_{-}(t)\right\rangle=0$, while $g_{\text {eff }}^{(2)} \neq 0$ such that $P_{e}(t) \neq 0$.

More generally, defining time-evolved dressed-state wave packets $f_{ \pm}(z, t)=\left\langle z\left|e^{-(i / \hbar) H_{ \pm} t} \int d z^{\prime} f_{0}\left(z^{\prime}\right)\right| z^{\prime}\right\rangle$ associated with $\left|\Psi_{\text {tot }}(0)\right\rangle$ leads to the excited-state probability for all times as

$$
P_{e}(t)=\frac{1}{2}+c_{+}^{*} c_{-} \operatorname{Re}\left(\int d z f_{+}^{*}(z, t) f_{-}(z, t)\right) .
$$

Here, $P_{e}(t)$ is given as an overlap (interference) of amplitudes for the dressed-state wave packets associated with the two potentials $V_{ \pm} \equiv \pm \hbar g(z)$. Significantly, coherent internal state dynamics are now linked self-consistently with the coherent evolution of the external c.m. state. A qualitatively similar linkage has been analyzed in the context of the micromaser operating with cold atoms [18].

The c.m. state dependency of these various expressions is made explicit by assuming a standing-wave cavity with $g(z)=g_{0} \cos (k z)$ and an infinite square well for $V_{\text {ext }}$,

$$
V_{\mathrm{ext}}=\left\{\begin{array}{cc}
\infty, & z<z_{1}, z>z_{1}+a \\
0, & z_{1}<z<z_{1}+a,
\end{array}\right.
$$

with $a \sim \lambda_{0} / 2 \pi=1 / k$, which has bound states

$$
\left\{\psi_{q}(z)\right\}=\left\{\sqrt{\frac{2}{a}} \sin \left(\frac{q \pi}{a}\left(z-z_{1}\right)\right)\right\}
$$

for $z_{1}<z<z_{1}+a$ and integers $q \geqslant 1$. The bound state energies are $\left\{E_{q}\right\}=\left\{\hbar^{2} \pi^{2} q^{2} / 2 m_{a} a^{2}\right\}$. Figure 3 illustrates the situation for the two bound states $q=1,2$.

The quadratic short time dependence of $P_{e}(t)$ with characteristic curvature given by the c.m. state-dependent rate $g_{\text {eff }}^{(2)}$ of Eq. (8) for the initial c.m. states $q=1,2$ is shown in Fig. 4. The dependence on $q$ of these curves can be understood from the difference in the overlap integrals of $\psi_{q}(z)$ with $|g(z)|^{2}$ as predicted by Eq. (8).

Turning now to dynamics on longer time scales, in Figs. 5 and 7 , we illustrate various aspects of the interplay of the c.m. and internal dynamics for several choices of the parameters $\left(m_{a}, g_{0}, R_{1}\right)$ and $f_{0}(z)$, where in all cases $c_{ \pm}= \pm 1 / \sqrt{2}$. From the insets in Fig. 5, $f_{0}(z)$ is taken to be either the ground state $\left[\psi_{1}(z)\right]$ or the first excited state $\left[\psi_{2}(z)\right]$ of $V_{\text {ext }}$. It is clear that for the choices of $z_{1}$ shown, the wave packets $f_{ \pm}(z, t)$ will move into adjacent potential wells because the dressed states $\left|D_{1, \pm}\right\rangle$ see potentials $V_{ \pm} \equiv \pm \hbar g_{0} \cos (k z)$. More quantitatively, $f_{ \pm}(z, t)$ are computed by reexpressing $f_{0}(z)$ in terms of the well-dressed states $\left\{\phi_{p}(z), E_{p}=\hbar \omega_{p}\right\}$ as

$$
f_{ \pm}(z, t)=\sum_{p} c_{p} e^{-i \omega_{p} t} \phi_{p}(z)
$$

where, as expected, the expansion coefficient is given by

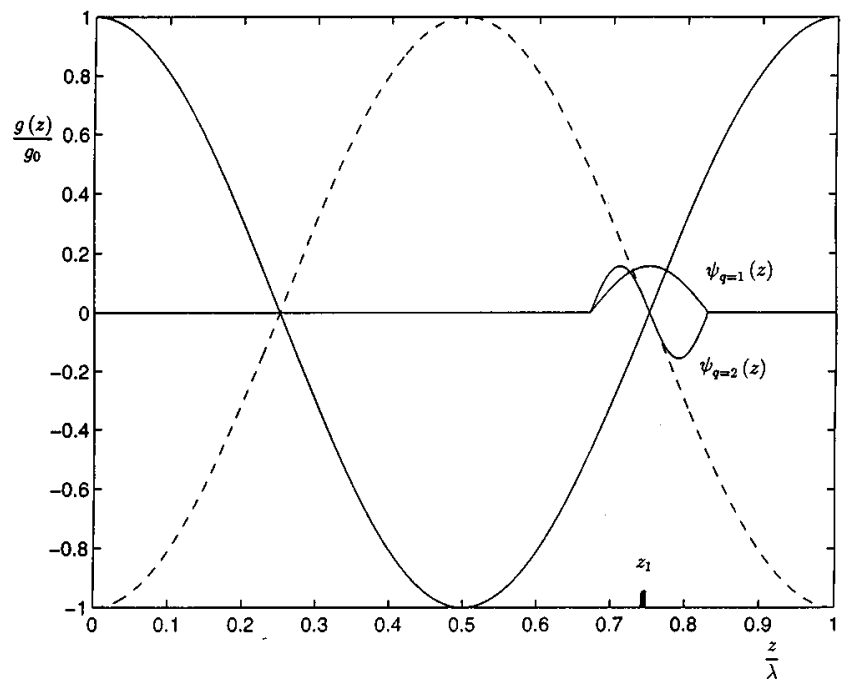

FIG. 3. An illustration of the situation in which bound states $\psi_{q=1,2}$ [Eq. (11)] of $V_{\text {ext }}(z)$ [Eq. (10)] evolve dynamically when starting in the atomic ground state in the one-photon field of a cavity with mode function $g(z)=g_{0} \sin (k z)$. The dressed states $\left|D_{1, \pm}\right\rangle$ will see the potentials labeled by $V_{+}$(dashed) and $V_{-}$ (solid), respectively.

$$
c_{p}=\int d z^{\prime} \phi_{p}^{*}\left(z^{\prime}\right) f_{0}\left(z^{\prime}\right)
$$

It is worth reiterating that in order to distinguish $f_{ \pm}(z, t)$ in Eq. (12), the appropriate $\left\{\phi_{p}(z)\right\}$ to use is the set localized to the (spatially distinct) potential minima seen by $\left|D_{1, \pm}\right\rangle$. Taking $g_{0} / 2 \pi=20 \mathrm{MHz}$ [as is relevant to Fig. 5(a)], for example, the potential $V_{+}=\hbar g(z)$ for $n=1$ is found numerically to have 177 bound states. For the initial states here with $a \ll \lambda_{0}$, we have verified the validity of evaluating the time evolution in terms of $\left\{\phi_{p}(z)\right\}$ only (i.e., neglecting the con-

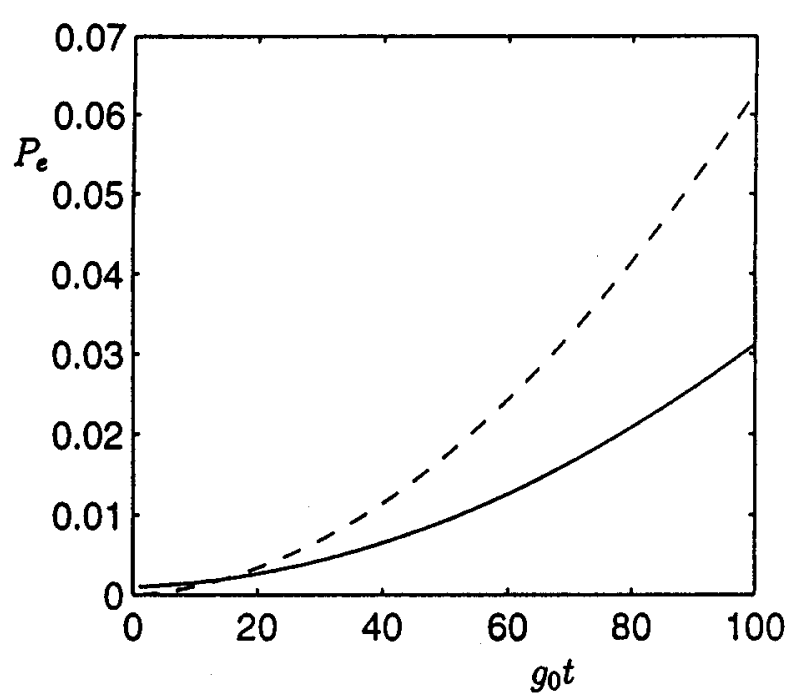

FIG. 4. Quadratic short time evolution of $P_{e}(t)$ for the two states $q=1$ (solid) and $q=2$ (dashed) of Fig. 3. The different rates of growth here are due to the overlap of these two wave functions with the quantum field profile $g(z)$ as quantified by $g_{\text {eff }}^{(2)}$ of Eq. (8). 

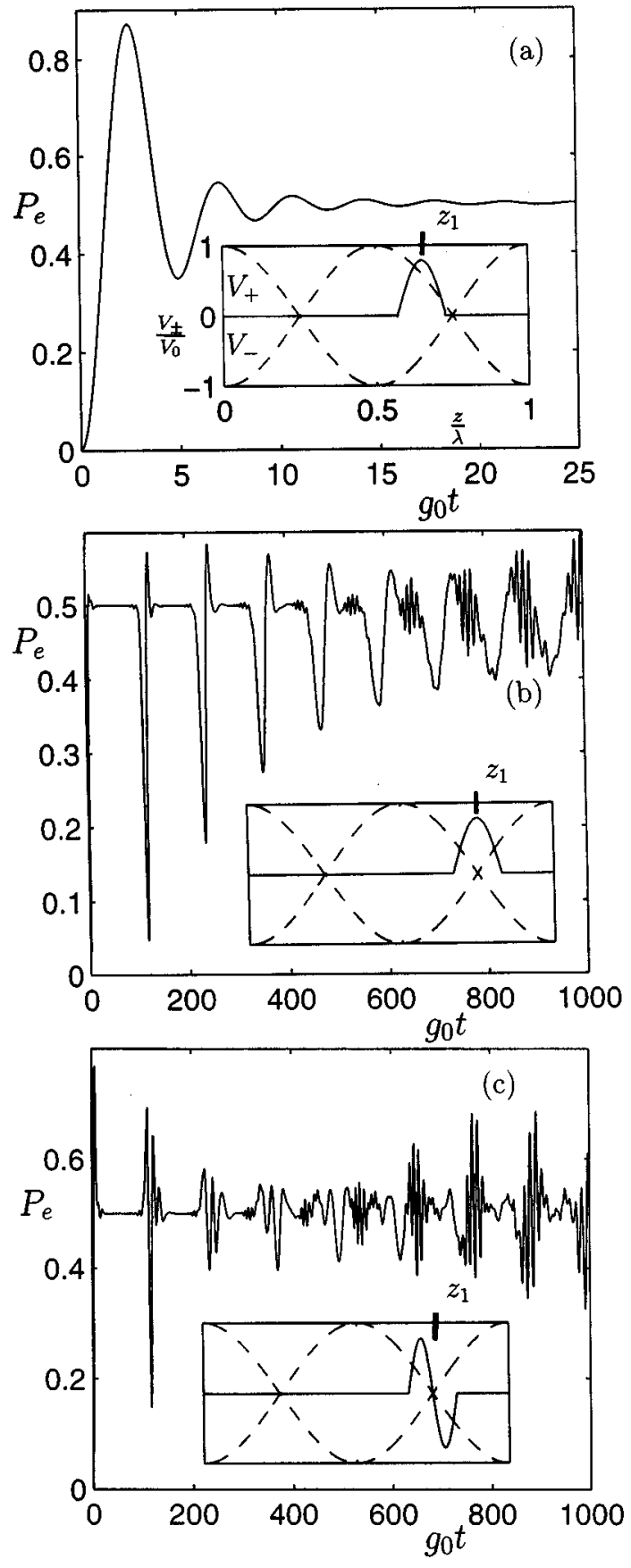

FIG. 5. Evolution of $P_{e}(t)$ for three different initial c.m. wave packets in the $n=1$ manifold with $m_{a}=133 \mathrm{amu}$. In (a) $g_{0} / 2 \pi=20 \mathrm{MHz}$ and $R_{1}=1 \times 10^{-4}$, while in (b) and (c) $g_{0} / 2 \pi=1 \mathrm{MHz}$ so that $R_{1}=2 \times 10^{-3}$. Insets show $f_{0}(z)$ (solid line) for the $q=1$ state in (a),(b) and $q=2$ in (c), and the (normalized) dressed-state potentials $V_{ \pm}$(dashed lines).

tinuum) with independent calculations using the split operator method [33]. In Fig. 6, we show the decomposition of the initial states $q=1,2$ in terms of the set of 30 bound states of $V_{+}$for the parameters of Figs. 5(b), and 5(c). In this figure, we plot $\left|c_{p}\right|^{2}$ from Eq. (13) as a function of bound state $p$. It is clear that, even neglecting the continuum, this basis is completely adequate for the decomposition of the states $\left\{\psi_{q}(z)\right\}$ for small $q$, and that the use of a more general basis (such as the delocalized Bloch states) would only obscure the

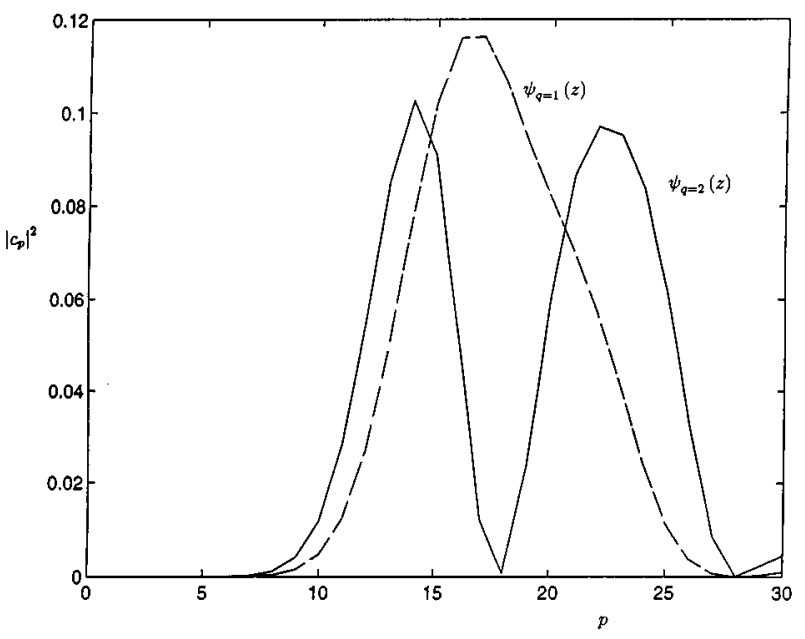

FIG. 6. Decomposition of the states $q=1$ (dashed) and $q=2$ (solid) of Fig. 3 in terms of the 30 bound states $\left\{\phi_{p}(z)\right\}$ of $V_{ \pm}$as is relevant to the parameters of Figs. 5(b) and 5(c). We plot $\left|c_{p}\right|^{\overline{2}}$ from Eq. (13) as a function of bound state $p$. In both cases, $\Sigma_{p}\left|c_{p}\right|^{2}>98 \%$.

dynamics. Of course, as $q$ increases, it would then become important to extend the basis over multiple wavelengths to account for tunneling of these higher energy states.

From Fig. 5(a), we see a time dependence for $P_{e}(t)$ reminiscent of the well-known "collapse" of Rabi oscillations caused by a spread in values $\Omega_{n}=2 g \sqrt{n}$ associated with a distribution in $n$ [34]. Similarly, here the collapse is brought about in part by dispersion in $\Omega_{1}$ (now for fixed $n=1$ ) set by the structure of $f_{0}(z)$ relative to $g(z)$. In addition, on a time scale $\tau_{1}$ given by

$$
g_{0} \tau_{1} \sim \sqrt{\frac{k a}{2 R_{1}}}
$$

the wave packets $f_{ \pm}(z, t)$ separate due to their evolutions along $V_{ \pm}$, driving the overlap of amplitudes in Eq. (9), and hence dynamical evolution of $P_{e}(t)$ to zero. A completely analogous evolution occurs for an initially excited atom with no photons in the cavity, which might be termed a "vacuumRabi" splitting [34] where now the atomic wave packet is split by its own radiation reaction field.

The longer term evolution of $P_{e}(t)$ is illustrated in Figs. $5(\mathrm{~b})$ and $5(\mathrm{c})$ for different choices of $f_{0}(z)$. Without delving into the details, it should be clear that there is a profound dependence of the dynamics on $f_{0}(z)$. For example, the initial rise of $P_{e}(t)$ for small $t$ is given by $g_{\text {eff }}^{(2)}$ in each case, but with a ratio $g_{\text {eff }}^{(2)}[q=1] / g_{\text {eff }}^{(2)}[q=2] \simeq 1.4$. Further note that $g_{\text {eff }}^{(1)}[q=1,2]=0$ in Figs. 5(b) and 5(c) while $g_{\text {eff }}^{(1)}[q=1] \neq 0$ in Fig. 5(a) as a consequence of the different initial c.m. states.

Beyond the initial "collapse" regime, $P_{e}(t)$ is quiescent with then a "revival" occurring near $g_{0} \tau_{2}=110$ as in Figs. 5(b) and 5(c). From the previous discussion of Fig. 1(c), an estimate of the "oscillator" frequency for the c.m. is $\mathcal{V}_{1} / g_{0} \sim \sqrt{2 R_{1}} \sim 2 \pi / 100$. Clearly, the overlap integral between $f_{ \pm}(z, t)$ in $P_{e}(t)$ in Eq. (9) has nonzero contributions at $z \sim z_{1}$ when the two dressed-state components of the wave packet interfere, connecting the c.m. time scale $2 \pi / \mathcal{V}_{1}$ to the 

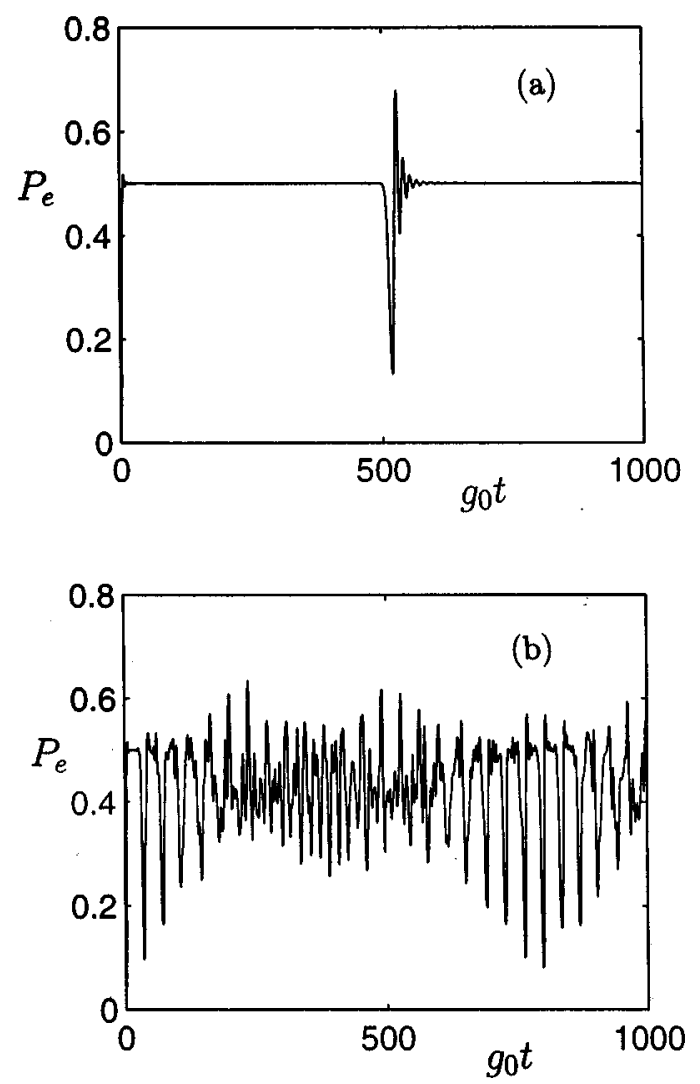

FIG. 7. The dependence of $P_{e}(t)$ on the ratio $R_{n}$ is illustrated. (a) Evolution of $P_{e}(t)$; here, $R_{1}=1 \times 10^{-4}$, with $m_{a}=133 \mathrm{amu}$ and $g_{0} / 2 \pi=20 \mathrm{MHz}$ as appropriate to the $D 2$ line in cesium at $852 \mathrm{~nm}$. In (b) $R_{1}=2 \times 10^{-2}$ with $g_{0} / 2 \pi=2.5 \mathrm{MHz}$ and $m_{a}=4$ amu for the transition at $1.083 \mu \mathrm{m}$ in $\mathrm{He}^{*}$.

long-term dynamical evolution of $P_{e}(t)$ via $\tau_{2}$. In fact, the resulting identification of $\tau_{2}$ as

$$
g_{0} \tau_{2} \sim \frac{2 \pi}{\sqrt{2 R_{1}}}
$$

produces reasonable agreement with $\tau_{2}$ in Figs. 5(b) and $5(\mathrm{c})$.

The particular details of the fine structure of $P_{e}(t)$ at multiples of the initial revival time $\tau_{2}$ (i.e., $\tau_{2}, 2 \tau_{2}, \ldots$ ) depend upon the overlap of $f_{0}(z)$ with $\left\{\phi_{p}(z)\right\}$. In qualitative terms, the oscillations for $q=1$ have twice the frequency as for $q=2$ since the probability density for $\psi_{2}(z)$ has twice the number of maxima as $\psi_{1}(z)$, illustrating the dependence of the dynamics on the initial c.m. state $q$. Beyond the first few revivals, the anharmonic character of $V_{ \pm}$enters to disperse the wave packet.

To illustrate further the interplay of internal atom-cavity and external c.m. time scales, we present in Fig. 7 two examples which emphasize the role of the parameter $R_{n}$. Since the characteristic frequency interval for the bound states of $g(z)$ given by $\mathcal{V}_{n} / g_{0} \sqrt{n} \sim \sqrt{2 R_{n}}, R_{n}$ should set the separation of time scales between the internal and external state dynamics, as Fig. 7 indeed demonstrates. Displayed is the quantity $P_{e}(t)$ from Eq. (9) with $c_{ \pm}= \pm 1 / \sqrt{2}$ and with $f_{0}(z)$ as in Fig. 5(b). Figure 7(a) is computed for $R_{1}=1 \times 10^{-4}$.
Figure 7(a) is representative of a rather large well depth $\left(g_{0} / 2 \pi=20 \mathrm{MHz}\right)$ and small energy spacing of the eigenvalues $\left\{E_{p}\right\}$ as for an optical transition in a heavy atom such as cesium, whose $D 2$ transition wavelength $\lambda_{0}=852 \mathrm{~nm}$ and mass $m_{a}=133 \mathrm{amu}$. On the other hand, Fig. 7(b) has $R_{1}=2 \times 10^{-2}$, corresponding to a shallow well $\left(g_{0} / 2 \pi\right.$ $=2.5 \mathrm{MHz}$ ) with a less pronounced separation of time scales between internal and external state dynamics. In this case, the parameters for a light atom, such as the metastable transition at $1.08 \mu \mathrm{m}$ in $\mathrm{He}^{*}$ of mass $m_{a}=4 \mathrm{amu}$ are appropriate.

Note from Fig. 7(b) that in addition to the revivals themselves at $\tau_{2}$ associated with the c.m. oscillations, there is yet a much larger time, $\tau_{3}$, for the recurrence of the revivals around $g_{0} \tau_{3}=800$ that arise because of the small size of the basis set $\left\{\left|\phi_{p}\right\rangle\right\}$, which in Fig. 7(b) contains only the bound states necessary for an accurate decomposition of $f_{0}(z)$. In this case, the potential wells $V_{ \pm}$contain only 12 bound states and the decomposition of the initial state $f_{0}(z)$ uses only about 5 of these. If the potential well were harmonic with evenly spaced energy eigenstates, for example, the decomposition of the initial c.m. state into eigenstates of the well could be considered like a Fourier series with only a finite number of components. In this case, the whole c.m. state time evolution is periodic at the highest eigenfrequency (Fourier component) in this c.m.-state decomposition. This is the physical origin of the time scale $\tau_{3}$. Note that although we have employed only the $n=1$ manifold in Figs. 5 and 7 , even more complex dynamics would follow from superpositions of other $n$ manifolds.

Beyond the context of our current work, such behavior is familiar, for example, in the dynamics of wave packets formed from sums of Rydberg states [35]. A distinguishing characteristic within the setting of cavity QED is the possibility for modifications of the external state potential via the internal state dynamics, and conversely. In fact, the inextricable interweaving of the atomic c.m. motion and the evolution of the cavity field via coupling to the atomic dipole leads to a host of exciting new phenomena at the frontier of quantum measurement [9].

\section{DISSIPATION}

Although we are currently investigating avenues of decoherence due to atomic spontaneous emission at rate $\Gamma$ and cavity decay at rate $\kappa$ rigorously via the QMCWF picture (with initial results reported in [31]), here we opt for a few qualitative comments. As a realistic example of Fig. 1(c), consider the $2 S \rightarrow 2 P$ transition at $1.083 \mu \mathrm{m}$ in $\mathrm{He}^{*}$ for which $\gamma_{\perp} / 2 \pi=\Gamma / 4 \pi \sim 800 \mathrm{kHz}$. When coupled to a FabryPérot microcavity with $g_{0} / 2 \pi \sim 120 \mathrm{MHz}$ and $\kappa / 2 \pi$ $\sim 2.9 \mathrm{MHz} \quad$ (length $=12 \mu \mathrm{m}$, finesse $=2 \times 10^{6} \quad[36]$ ), this gives $\mathcal{V}_{n} / 2 \pi \sim 3.3 \mathrm{MHz}>\left(\gamma_{\perp}, \kappa\right) / 2 \pi$ which opens up the possibility for experimental verification of modification to the Jaynes-Cummings manifold due to the quantized c.m. even in the presence of dissipation. An optical microsphere of $\sim 20 \mu \mathrm{m}$ diameter has $g_{0} / 2 \pi \sim 125 \mathrm{MHz}$ and $\kappa / 2 \pi$ $\sim 100 \mathrm{kHz}$ for a reasonable $Q \sim 10^{9}$, which would again allow resolution of the well-dressed state splitting of Fig. 1(c) even for the $n=1$ manifold. In fact, $g_{0} \sim 10^{4} \kappa$ seems feasible for microspheres [30,37]. Generally in the optical domain, 
$g_{0} / \Gamma \leqslant 10^{2}$, although the role of $\Gamma$ can be greatly mitigated through the use of dark-state resonances [19]. By contrast, present technology in the microwave domain has already achieved $\quad g_{0} / \kappa, \Gamma>10^{3}-10^{4} \quad[1], \quad$ albeit with $\left[\left(\hbar g_{0} / k_{B}\right) \sim 1 \mu \mathrm{K}\right] \ll E_{k} / k_{B}$.

With regard to recoil kicks from atomic decay, note that significant heating of the c.m. wave packet requires a time $T_{H} \sim\left(\Delta E_{q} / E_{\text {recoil }}\right) \Gamma^{-1}$ [31], where $\Delta E_{q}$ is the level spacing of the relevant well-dressed states (Fig. 1) and $E_{\text {recoil }}=\hbar^{2} k^{2} / 2 m_{a}$. As an example, for the case of Fig. 1(c), with $\xi \sim \lambda_{0} / 2 \pi, g_{0} T_{H} \sim\left(g_{0} / \Gamma\right)\left(1 / \sqrt{R_{n}}\right) \gg g_{0} / \Gamma$ for $R_{n}$ small as in Figs. 5 and 7. Here, the role of $R_{n}$ is to increase the naive estimate for the recoil heating time of $\Gamma^{-1}$ by a factor $\sim 1 / \sqrt{R_{n}}$ by imposing bound state structure on the final c.m. state in a spontaneous emission recoil.

\section{CONCLUSIONS}

In conclusion, we have investigated the marriage of cavity QED with the atomic c.m. wave function and have analyzed some of the remarkable progeny that spring from this union. The "well-dressed" states with the spectrum of eigenvalues illustrated in Fig. 1 replace the more familiar dressed states of the Jaynes-Cummings Hamiltonian, with three experimen- tally distinguishable regimes having been identified. Implicit in the structure of the well-dressed states are modifications of the usual interpretation of the atom-field coupling $g(\mathbf{r})$, such as the quantities $g_{q q}$, which express the overlap of the $g(\mathbf{r})$ with the bound states $\psi_{q}(\mathbf{r})$ or the quantities $\left\{E_{p}, \phi_{p}(\mathbf{r})\right\}$, which describe the bound state structure associated with the spatial variation of $g(\mathbf{r})$ itself.

In addition, qualitative modifications of the usual dynamical processes in cavity QED arise from the interweaving of internal (atomic dipole+cavity field) and c.m. degrees of freedom. Although these ideas have been illustrated with reference to a specific example (as in Figs. 5 and 7), we anticipate as well that many features of our dynamical analysis (e.g., the time scales $\tau_{i}$ and couplings $g_{\text {eff }}^{(i)}$ ) will be generic to more realistic systems with 3D geometries and dissipation.

\section{ACKNOWLEDGMENTS}

This work was funded by the Office of Naval Research, the National Science Foundation, and DARPA through the QUIC program (Quantum Information and Computation) administered by the U.S. Army Research Office. D.W.V. acknowledges financial support from NSERC.
[1] See, e.g., Cavity Quantum Electrodynamics, Advances in Atomic, Molecular and Optical Physics, Supplement 2, edited by P. R. Berman (Academic, San Diego, 1994).

[2] H. Mabuchi, Q. A. Turchette, M. S. Chapman, and H. J. Kimble, Opt. Lett. 21, 1393 (1996).

[3] B. G. Englert, J. Schwinger, A. O. Barut, and M. O. Scully, Europhys. Lett. 14, 25 (1991).

[4] S. Haroche, M. Brune, and J. M. Raimond, Europhys. Lett. 14, 19 (1991).

[5] B. W. Shore, P. Meystre, and S. Stenholm, J. Opt. Soc. Am. B 8, 903 (1991).

[6] T. W. Mossberg, M. Lewenstein, and D. J. Gauthier, Phys. Rev. Lett. 67, 1723 (1991).

[7] A. C. Doherty, A. S. Parkins, S. M. Tan, and D. F. Walls, Phys. Rev. A 56, 833 (1997).

[8] H. Mabuchi, Quantum Semiclassic. Opt. 8, 1103 (1996).

[9] P. Storey, M. J. Collett, and D. F. Walls, Phys. Rev. Lett. 68, 472 (1992).

[10] A. M. Herkommer, H. J. Carmichael, and W. P. Schleich, Quantum Semiclassic. Opt. 8, 189 (1996).

[11] A. M. Herkommer, V. M. Akulin, and W. P. Schleich, Phys. Rev. Lett. 69, 3298 (1992).

[12] M. J. Holland, D. F. Walls, and P. Zoller, Phys. Rev. Lett. 67, 1716 (1991).

[13] I. S. Averbukh, V. M. Akulin, and W. P. Schleich, Phys. Rev. Lett. 72, 437 (1994).

[14] A. B. Matsko, S. P. Vyatchinin, H. Mabuchi, and H. J. Kimble, Phys. Lett. A 192, 175 (1994).

[15] F. Treussart, J. Hare, L. Collot, V. Lefèvre, D. S. Weiss, V. Sandoghar, J. M. Raimond, and S. Haroche, Opt. Lett. 19, 1651 (1994).
[16] Z. Byalnicka-Birula, P. Meystre, E. Schumaker, and M. Wilkens, Opt. Commun. 85, 315 (1991).

[17] W. Ren and H. J. Carmichael, Phys. Rev. A 51, 752 (1995).

[18] M. O. Scully, G. M. Meyer, and H. Walther, Phys. Rev. Lett. 76, 4144 (1996).

[19] T. Pellizzari, S. A. Gardiner, J. I. Cirac, and P. Zoller, Phys. Rev. Lett. 75, 3788 (1995).

[20] J. I. Cirac, P. Zoller, H. J. Kimble, and H. Mabuchi, Phys. Rev. Lett. 78, 3221 (1997).

[21] C. K. Law and H. J. Kimble, J. Mod. Opt. (to be published).

[22] C. K. Law and J. H. Eberly, Phys. Rev. Lett. 76, 1055 (1996).

[23] C. Monroe, D. M. Meekhof, B. E. King, S. R. Jefferts, W. M. Itano, D. J. Wineland, and P. Gould, Phys. Rev. Lett. 75, 4011 (1995).

[24] P. Marte, R. Dum, R. Taïeb, P. D. Lett, and P. Zoller, Phys. Rev. Lett. 71, 1335 (1993); Y. Castin and J. Dalibard, Europhys. Lett. 14, 761 (1994); M. Wilkens, E. Schumacher, and P. Meystre, Phys. Rev. A 44, 3130 (1991); M. BenDahan, E. Peik, J. Reichel, Y. Castin, and C. Salomon, Phys. Rev. Lett. 76, 4508 (1996); S. Wilkinson, C. Bharucha, K. Madison, Qian Niu, and M. Raizen, ibid. 76, 4512 (1996).

[25] J. I. Cirac, A. S. Parkins, R. Blatt, and P. Zoller, Opt. Commun. 97, 353 (1995).

[26] D. J. Wineland, J. Dalibard, and C. Cohen-Tannoudji, J. Opt. Soc. Am. B 9, 32 (1992); J. I. Cirac, R. Blatt, P. Zoller, and W. D. Phillips, Phys. Rev. A 46, 2668 (1992).

[27] R. J. C. Spreeuw, T. Pfau, U. Janicke, and M. Wilkens, Europhys. Lett. 32, 469 (1995); M. Holland, K. Burnett, C. Gardiner, J. I. Cirac, and P. Zoller, Phys. Rev. A 54, R1757 (1996).

[28] H. Wallis, J. Dalibard, and C. Cohen-Tannoudji, Appl. Phys. 
B 54, 407 (1992); M. Holland, S. Marksteiner, P. Marte, and P. Zoller, Phys. Rev. Lett. 76, 3683 (1996); J. Dalibard and C. Cohen-Tannoudji, J. Opt. Soc. Am. B 2, 1707 (1985).

[29] See, e.g., C. Cohen-Tannoudji, J. Dupont-Roc, and G. Grynberg, Atom-Photon Interactions (Wiley, New York, 1992), pp. $407 \mathrm{ff}$.

[30] H. Mabuchi and H. J. Kimble, Opt. Lett. 19, 749 (1994).

[31] D. W. Vernooy and H. J. Kimble, Phys. Rev. A 55, 1239 (1997).
[32] N. Davidson, H. J. Lee, M. Kasevich, and S. Chu, Phys. Rev. Lett. 72, 3158 (1994).

[33] R. Kosloff, J. Phys. Chem. 92, 2087 (1988).

[34] H. I. Yoo and J. H. Eberly, Phys. Rep. 118, 239 (1985).

[35] M. W. Noel and C. R. Stroud, Phys. Rev. Lett. 77, 1913 (1996).

[36] G. Rempe, R. J. Thompson, H. J. Kimble, and R. Lalezari, Opt. Lett. 17, 365 (1992).

[37] M. L. Gorodetsky, A. A. Savchenkov, and V. S. Ilchenko, Opt. Lett. 21, 453 (1995). 